\title{
Sequence Variations of Epstein-Barr Virus-Encoded Small Noncoding RNA and Latent Membrane Protein 1 in Hematologic Tumors in Northern China
}

\author{
Hai-Yu Wang ${ }^{a, b}$ Lingling Sun ${ }^{c}$ Ping Lid ${ }^{d}$ Wen Liu ${ }^{a}$ Zhong-Guang Zhang ${ }^{a}$ \\ Bing Luo ${ }^{a}$ \\ aDepartment of Pathogenic Biology, Qingdao University Medical College, Qingdao, China; ${ }^{b}$ Department of \\ Infection-Control, The First People's Hospital of Lianyungang, Lianyungang, China; 'Department of Pathology, \\ The Affiliated Hospital of Qingdao University, Qingdao, China; ${ }^{d}$ Department of Blood Transfusion, \\ The Affiliated Hospital of Qingdao University, Qingdao, China
}

\section{Keywords}

Epstein-Barr virus · Hematologic tumors - Genotype ·

Epstein-Barr virus-encoded small noncoding RNA · Latent membrane protein $1 \cdot$ Polymorphism

\begin{abstract}
Objective: To investigate the relationship between hematologic tumors and Epstein-Barr virus (EBV)-encoded small noncoding RNA (EBER) variations as well as latent membrane protein 1 (LMP1) variations. Methods: Patients with leukemia and myelodysplastic syndrome (MDS) were selected as subjects. Genotypes $1 / 2$ and genotypes F/f were analyzed using the nested PCR technology, while EBER and LMP1 subtypes were analyzed by the nested PCR and DNA sequencing. Results: Type 1 was more dominant than type 2, found in 59 out of 82 (72\%) leukemia and in 31 out of 35 (88.6\%) MDS, while type $F$ was more prevalent than type $f$ in leukemia $(83 / 85,97.6 \%)$ and MDS $(29 / 31,93.5 \%)$ samples. The distribution of EBV genotypes 1/2 was not significantly different among leukemia, MDS, and healthy donor groups, neither was that of EBV genotypes F/f. EB-6m prototype was the dominant subtype of EBER in leukemia and MDS (73.2\% [30/41] and 83.3\% [10/12], respectively). The frequency of
\end{abstract}

EB-6m was lower than that of healthy people $(96.7 \%, 89 / 92)$, and the difference was significant $(p<0.05)$. China 1 subtype was the dominant subtype of LMP1 in leukemia and MDS (70\% [28/40] and 90\% [9/10], respectively), and there was no significant difference in the distribution of LMP1 subtypes among the 3 groups $(p>0.05)$. Conclusion: The distribution of EBV 1/2, F/f, EBER, and LMP1 subtypes in leukemia and MDS was similar to that in the background population in Northern China, which means that these subtypes may be rather region-restricted but not associated with leukemia and MDS pathogenesis.

๑) 2021 S. Karger AG, Basel

\section{Introduction}

Epstein-Barr virus (EBV) is recognized as a tumor virus that is closely related to the development of multiple tumors, such as nasopharyngeal carcinoma (NPC), gastric cancer, Hodgkin's lymphoma, natural killer T-cell lymphoma, and others [1-5]. In recent years, many studies have focused on the relationship between leukemia and EBV. The unique pattern of EBV expression can be considered as a marker of the particular differentiation win-

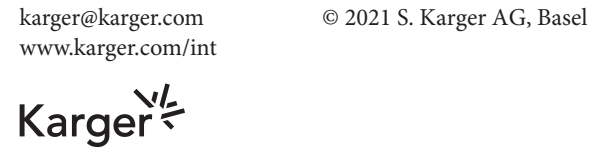

Zhong-Guang Zhang

Department of Pathogenic Biology, Qingdao University Medical College 38 Dengzhou Road

Qingdao 266021 (China)

zhangzhongguang@126.com 
dow of the B cell, which can result in chronic leukemia [6]. Ateyah et al. [7] suggested that patients with B-cell acute lymphoblastic leukemia infected with EBV had a significant increase in the expression of CD4+CD25high+ Foxp3+Treg. Several previous investigations have been carried out focusing on the relationship between EBV infection and later leukemogenesis, which seems to play a vital role in the development of ALL [8-11]. Ahmed et al. [9] reported that although EBV infection has no theoretical link with the pathogenesis of AML (EBV infects B lymphocytes), EBV latent membrane protein 1 (LMP1) gene transcripts were found in 5 of 15 (33.3\%) patients with AML. EBV-DNA copy numbers were discovered in 19 of 75 (25.3\%) individuals with AML [8]. It is possible that EBV infection, as a secondary event, could increase the risk of AML. It is also reported that EBV infection is a possible factor in the progression of CLL $[12,13]$. EBV-DNA load in CLL is an independent predictor of clinical course and survival [14]. However, the potential role of EBV in these hematologic tumors still remains unclear.

Many EBV subtypes have been found in the study of EBV gene polymorphism, but the relationship between EBV-coding gene variations and tumorigenesis is still unclear. EBV is classified as genotypes 1 and 2 (also called A and $B$ ) on the basis of the sequence divergence within EBV nuclear antigens 2 and 3 (EBNA2 and EBNA3, respectively) [15-17]. When further studies were performed to investigate other polymorphisms of EBV, type F/f was determined. Genotype F/f is assigned according to the presence or absence of the BamHI restriction site. PCR and restriction fragment length polymorphism were employed for distinguishing prototype $\mathrm{F}$ and $\mathrm{f}$ variants, as described previously $[18,19]$.

EBV-encoded small RNAs (EBERs, EBER1 and EBER2) are the most abundant EBV transcripts in EBV-associated tumors [20, 21]. It has long been believed that the coding sequences of EBERs are conserved among various EBV isolates. Therefore, mutations in the EBER genes and their relationship with EBV-associated diseases have drawn little attention. Three major subtypes of EBER (EB-6m, EB$8 \mathrm{~m}$, and EB-10m) were identified. LMP1 plays crucial roles in the development of EBV-related carcinomas, comprising a short $\mathrm{N}$-terminal cytoplasmic tail of 17 amino acids, 6 hydrophobic transmembrane segments, and 1 long Cterminal cytosolic region of 200 amino acids $[22,23]$. The variation of LMP1 can be distinguished by the variation of $\mathrm{C}$-terminal sequence and $\mathrm{N}$-terminal sequence, and considerable sequence variations were detected in the C-terminal region. Initially, Hu et al. [24] found XhoI (-) mutation (the loss of XhoI sites in the N-terminal first exon) in the Chinese NPC strains CAO. Miller et al. [25] found a deletion from amino acids 343 to 352 in the C-terminal (del-LMP1 variation) in Chinese EBV strains. Subsequent studies have shown that there are more variations in LMP1, including base mutations, other deletions such as $69 \mathrm{bp}$ deletions, and changes in the number of repeat sequences [26]. In a recent research by Edwards et al. [27], LMP1 was divided into 7 distinct sequence patterns or strains: B95-8, China 1, China 2, China 3, Med (Mediterranean), NC (North Carolina), and Alaskan on the basis of the amino acid (aa) changes of the carboxyl-terminal sequence (aa187-386) from different geographical origins.

We have detected EBER and LMP1 gene variations in various samples including samples from gastric carcinomas, NPCs, and lymphomas, as well as samples from healthy donors [28-31]. However, the variations of these EBV-coding genes in hematological diseases have not been found. Despite the ubiquity of EBV infection, the incidence of EBV-associated neoplasms is low and varies in different geographic regions. Considering the different geographical distribution of EBV subtypes, some suggested that they are only region-restricted, while others presented disease-specific evidences. To find EBV gene variations in more EBV-associated diseases in Northern China and try to give clues to the link between these mutations and EBV-related tumors, we decided to investigate sequence variations in the EBER and LMP1 and also compare these results with those from previous reports. The traditional EBNA2 variants (EBV type 1/type 2) and F/f type were also analyzed.

\section{Materials and Methods}

\section{Clinical Samples}

Peripheral blood specimens of 322 leukemia patients and 106 myelodysplastic syndrome (MDS) patients were obtained from the Affiliated Hospital of Qingdao University (from June 2017 to January 2018), which located in Shandong Province, Northern China. Data on healthy donors were adopted from our previous report, which were reported except for LMP1 [28, 31]. Four milliliters of the peripheral blood samples was collected and centrifuged at $1,200 \mathrm{rpm}$ for $10 \mathrm{~min}$. EDTA was used as an anticoagulant. The intermediate leukocyte layer was extracted, and the DNA was extracted by protease $\mathrm{K}$ digestion and phenol-chloroform method.

\section{Genotyping of EBV Genotypes $1 / 2$ and $F / f$}

Nested PCR was performed to analyze the EBV genotypes $1 / 2$ according to the sequence divergence within EBNA2, as previously reported [32]. Restriction fragment length polymorphism was performed to analyze genotypes F/f, as described by Cui et al. [33]. The sequences of primers used in the PCR and the product size of the corresponding genotypes are listed in Table 1. 
Table 1. Sequences of primers used in PCR

\begin{tabular}{|c|c|c|c|}
\hline Name of primers & Sequence $\left(5^{\prime}-3^{\prime}\right)$ & B95-8 coordinates & Size of PCR products \\
\hline \multicolumn{4}{|l|}{$1 / 2$ types } \\
\hline Type F1 & AGGGATGCCTGGACACAAG & & \\
\hline Type R1 & AGGGATGCCTGGACACAAG & & \\
\hline Type 1-1 & TCTTGATAGGGATCCGCTAGGATA & & Type $1=498$ bp \\
\hline Type 1-2 & ACCGTGGTTCTGGACTATCTGGAT & & \\
\hline Type 2-1 & CATGGTAGCCTTAGGACATA & & Type $2=150$ bp \\
\hline Type 2-2 & AGACTTAGTTGATGCCCTAG & & \\
\hline \multicolumn{4}{|l|}{ F/f types } \\
\hline Type F-1 & TCCCACCTGTTACCACATTC & & Type $F=198$ bp \\
\hline Type F-2 & GGCAATGGGACGTCTTGTAA & & Type $f=127+71 b p$ \\
\hline \multicolumn{4}{|l|}{ EBER } \\
\hline EBER-OS1 & AATGAGGGTTAGCATAGGC & $6,513-6,531$ & $718 \mathrm{bp}$ \\
\hline EBER-O1 & GTCACAGAATTGATTGGCA & $7,230-7,212$ & \\
\hline EBER-NS1 & GTCTGTCTTGAGGAGATGT & $6,585-6,603$ & $600 \mathrm{bp}$ \\
\hline EBER-N1 & TTTGTGTTGTAGGGGTAGC & $7,184-7,166$ & \\
\hline \multicolumn{4}{|l|}{$L M P 1$} \\
\hline LMP1-1 & TGAATGTGGCTTTTCAGCCTAGACA & $168,015-168,039$ & $1,613 \mathrm{bp}$ \\
\hline LMP1-6 & CCCGTACTGCCTCCGGCAGAC & $169,627-169,607$ & \\
\hline LMP1-3 & GTCATAGTAGCTTAGCTGAAC & $168,163-168,183$ & $668 \mathrm{bp}$ \\
\hline LMP1-4 & GTGGACTCTATTGGTTGATCTC & $168,830-168,808$ & \\
\hline LMP1-2 & CATCCCAAGAAACACGCGTT & $169,548-169,565$ & $772 \mathrm{bp}$ \\
\hline LMP1-5 & CAACCAATAGAGTCCACCAGT & $168,813-168,833$ & \\
\hline
\end{tabular}

EBER, Epstein-Barr virus (EBV)-encoded small noncoding RNA; LMP1, latent membrane protein 1.

Amplification of the EBER Gene and LMP1 Gene

We performed nested PCR to amplify a chosen region (nt6629nt7128) of the EBER gene and a chosen region (nt169474nt168163) of the LMP1 gene. The first PCR was carried out in a total volume of $25 \mu \mathrm{L}$ containing $1 \times$ PCR reaction buffer, $100 \mathrm{ng}$ of genomic DNA, $0.5 \mu \mathrm{M}$ of each primer, $200 \mu \mathrm{M}$ of each deoxyribonucleotide triphosphates, and $1 \mathrm{U}$ PfuTaq polymerase (TaKaRa Biotechnology Co., Ltd., Kyoto, Japan). PCR amplification was started with an initial denaturation at $94^{\circ} \mathrm{C}$ for $5 \mathrm{~min}$. Next, 38 cycles of denaturation at $94^{\circ} \mathrm{C}$ for $30 \mathrm{~s}$, annealing at $55^{\circ} \mathrm{C}$ for $30 \mathrm{~s}$, and extension at $72^{\circ} \mathrm{C}$ for $1 \mathrm{~min}$. A final elongation step at $72^{\circ} \mathrm{C}$ for 10 min was also conducted. Two microliters of the PCR product was used as the template DNA in the second-round PCR; the experimental condition of the second-round PCR is the same as the first round. The PCR products were analyzed by electrophoresis via $1.2 \%$ agarose gel.

We sent the amplified products to Beijing Genomics Institute for full-length sequencing. After sequencing, all samples were compared with the EBV prototype B95-8 (GenBank: V01555.2).

\section{Data Analysis}

Data were analyzed by SPSS 15.0 statistical software (SPSS, Chicago, IL, USA), and $p<0.05$ was considered statistically significant. The $\chi^{2}$ test and Fisher's exact tests were performed to determine the distribution difference of the EBV variations.

EBER and LMP1 Polymorphisms in the EBV-Associated Hematopathy

\section{Results}

\section{EBV-Positive Frequency in Leukemia and MDS} Samples

EBV-DNA copies were found in 88 of the 322 leukemia samples (27.3\%) and 35 of the 106 MDS samples $(33 \%)$.

\section{Genotypes of EBV in Leukemia and MDS Samples}

One hundred and twenty-three EBV-positive specimens, including 88 leukemia and 35 MDS samples, were analyzed. However, we failed to amplify the explored region of EBNA2 in 6 leukemia samples and failed to amplify the explored region of BamHI F in 3 leukemia samples as well as 4 MDS samples. We tried to change the annealing temperature of these samples, which failed to amplify, but the amplification failed. We speculated that the degradation or contamination of the specimens might contribute to the failure to amplify the explored region. The EBV genotype 1 was detected in 59 of the 82 leukemia specimens (72\%), 31 of the 35 MDS specimens 
Table 2. Distribution of EBV subtypes, EBER, and LMP1 variants in leukemia and MDS

\begin{tabular}{lccc}
\hline & Leukemia, $n(\%)$ & MDS, $n(\%)$ & $H, n(\%)$ \\
\hline EBNA2 (1/2) & & & \\
Type 1 & $59(72)$ & $31(88.6)$ & $45(78.9)$ \\
Type 2 & $7(8.5)$ & $3(8.6)$ & $10(17.6)$ \\
Type 1 + type 2 & $16(19.5)$ & $1(2.8)$ & $2(3.5)$ \\
\hline Total & $82(100)$ & $35(100)$ & $57(100)$ \\
\hline BamHI F (F/f) & & & \\
Type F & $83(97.6)$ & $29(93.5)$ & $98(94.2)$ \\
Type f & $2(2.4)$ & $2(6.5)$ & $6(5.8)$ \\
\hline Total & $85(100)$ & $31(100)$ & $104(100)$ \\
\hline EBER variants & & & \\
B95-8 & $11(26.8)$ & $2(16.7)$ & $1(1.1)$ \\
EB-6m & $30(73.2)$ & $10(83.3)$ & $89(96.7)$ \\
EB-8m & 0 & 0 & $1(1.1)$ \\
UG & 0 & 0 & $1(1.1)$ \\
\hline Total & $41(100)$ & $12(100)$ & $92(100)$ \\
\hline LMP1 variants & & & \\
B95-8 & $7(17.5)$ & $1(10)$ & 0 \\
China 1 & $28(70)$ & $9(90)$ & $30(69.8)$ \\
China 2 & $2(5)$ & 0 & $1(2.3)$ \\
Med- & $3(7.5)$ & 0 & $3(7)$ \\
RG & 0 & 0 & $43(100)$ \\
\hline Total & $40(100)$ & $10(100)$ & \\
\hline & & & \\
\hline
\end{tabular}

EBV, Epstein-Barr virus; EBER, EBV-encoded small noncoding RNA; LMP1, latent membrane protein 1; MDS, myelodysplastic syndromes; $H$, healthy donors.

(88.6\%), and 45 of 57 healthy donors (78.9\%). Seven of the 82 leukemia specimens (8.5\%), 3 of the 35 MDS specimens $(8.6 \%)$, and 10 of the 57 healthy donors $(17.6 \%)$ were defined as EBV genotype 2. Sixteen of the leukemia samples (19.5\%) and 1 of the MDS samples (2.8\%) as well as 2 of the healthy donors (3.5\%) carried both genotypes (type 1 and type 2) (Table 2). Genotypes $\mathrm{F}$ and $\mathrm{f}$ were found in $83(97.6 \%)$ and $2(2.4 \%)$ of the leukemia samples, respectively, while genotypes $\mathrm{F}$ and $\mathrm{f}$ were found in $29(93.5 \%)$ and $2(6.5 \%)$ of the MDS samples, respectively, and genotypes $\mathrm{F}$ and $\mathrm{f}$ were found in $98(94.2 \%)$ and $6(5.8 \%)$ of the healthy donors samples, respectively (Table 2).

There was no significant difference in the distribution of EBV genotypes $1 / 2$ and $\mathrm{F} / \mathrm{f}$ among the 3 groups $(p>$ $0.05)$. Data on healthy donors were adopted from our previous report [30,31].

\section{Sequence Variations of EBER Gene}

The EBER gene was amplified successfully in 41 leukemia samples and 12 MDS samples. We compared the obtained EBER sequences with the prototype B95-8 sequence. The sequence variations are shown in Figure 1. One sequence was chosen to represent all the samples with the same sequence in the figure. Two of the previously described subtypes [31] were found in these cases: EB-6m and B95-8. However, none of the EB-8m or EB$10 \mathrm{~m}$ subtypes were detected.

The B95- 8 subtype had the same EBER sequence as the EBV standard strain B95-8 or only appeared random mutations, as shown in Figure 1. B95-8 was found in 13 isolates, including 11 of the 41 leukemia specimens (26.8\%) and 2 of the 12 MDS specimens (16.7\%) (Table 2).

EB-6m was detected in 40 isolates, including 30 of the 41 leukemia samples (73.2\%) and 10 of the $12 \mathrm{MDS}$ samples (83.3\%). Common changes were found at the following base pair positions: 7123nt $\mathrm{A} \rightarrow \mathrm{G}$ in EBER2, 6808nt T $\rightarrow$ A, 6884nt G $\rightarrow$ A, 6886nt T $\rightarrow$ G, 6911nt A $\rightarrow \mathrm{G}$, and 6944nt $\mathrm{G} \rightarrow \mathrm{A}$ in the intergenic region. However, 1 leukemia sample had only 5 common mutations of EB-6m (6808nt, 6884nt, 6911nt, 6944nt, and 7123nt). In addition to the 6 common mutations, other sporadic mutations were found in some specimens shown in Figure 1.

The distribution of EBER subtypes between leukemia and MDS samples was not significantly different $\left(\chi^{2}=\right.$ $0.114, p=0.735)$. However, the distribution of EBER variations between leukemia, MDS, and healthy donors was different, and the difference was statistically significant $\left(\chi^{2}=15.757, p=0.000\right)$. The detection rate of EB-6m in leukemia and MDS was significantly lower than that in healthy people.

\section{Sequence Variations of LMP1 Gene}

Figure 2 shows the comparison results of LMP1 sequence variations and the prototype B95- 8 sequence. But it shows only the common amino acid changes, while all the amino acid changes are shown in supplementary information (online suppl. Fig. 1; for all online suppl. material, see www.karger.com/doi/10.1159/000510398). Four distinct sequence patterns were found in the specimens: B95-8, China 1, China 2, and Med (Mediterranean). Forty of the 88 leukemia samples and 10 of the 35 MDS samples were amplified in the LMP1 gene successfully. China $1(74 \%, 37 / 50)$ was the predominant subtype. There was no significant difference in the distribution of LMP1 subtypes between the 2 groups $(p>0.05)$. The results are summarized in Table 2. 


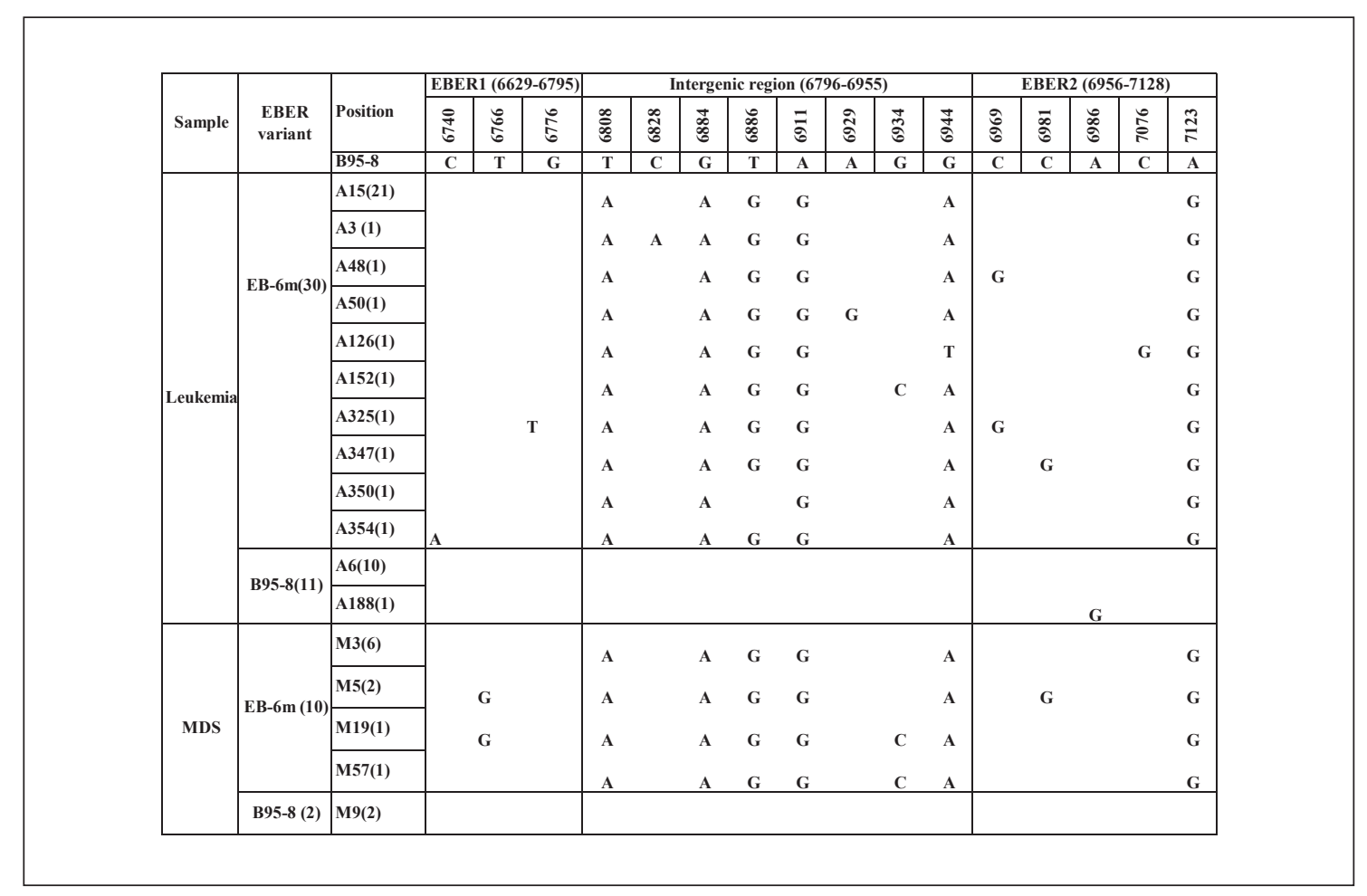

Fig. 1. EBER variations in leukemia and MDS in northern China The numbers across the second row correspond to nucleotide positions. The B95-8 nucleotide sequence is listed under the second row. Different subtypes are noted in the second column; however, isolates showing identical sequence within each population group are listed only by a representative isolate in the third column. The numbers in the third column refer to these representative isolates, and the capital letter A represents leukemia specimen, while the capital letter M represents MDS specimen. The numbers in parentheses after the specimen represent the number of specimens. The capital letters denote the nucleotides. Some rare sporadic mutations are not shown. EBER, Epstein-Barr virus (EBV)-encoded small noncoding RNA; MDS, myelodysplastic syndrome.

\section{B95-8}

The B95-8 subtype had the same sequence as the EBV standard strain B95-8 or only appeared random mutations, as shown in Figure 2. Seven of the 40 leukemia samples (17.5\%) and 1 of the 10 MDS samples (10\%) carried the B95- 8 subtype.

\section{China 1}

The China 1 subtype was detected in $70 \%$ of the leukemia samples (28/40) and $90 \%$ of the MDS samples (9/10). Forty common changes were found in the sequences, including 24 common coding changes: 3 (His $\rightarrow$ Arg), 13 (Arg $\rightarrow$ Pro), 17 (Arg $\rightarrow$ Leu), 25 (Leu $\rightarrow$ Ile), 46 (Asp $\rightarrow$ Asn), 82 (Ala $\rightarrow$ Gly), 84 (Cys $\rightarrow$ Gly), 85 (Ile $\rightarrow$ Leu), 106 (Phe $\rightarrow$ Ile), 122 (Ile $\rightarrow$ Leu), 126 (Leu $\rightarrow$ Phe), 129 (Met $\rightarrow$ Ile), 144 (Phe $\rightarrow$ Ile), 150 (Asp $\rightarrow$ Ala), $151($ Leu $\rightarrow$ Ile), 178 (Leu $\rightarrow$ Met), 189 (Pro $\rightarrow$ Gln), 192 (Ser $\rightarrow$ Thr), 212 (Gly $\rightarrow$ Ser), 309 (Ser $\rightarrow$ Asn), 322 (Gln $\rightarrow$ Asn), 334 (Gln $\rightarrow$ Arg), 338 (Leu $\rightarrow$ Ser), and 366 (Ser $\rightarrow$ Thr). Further- more, 16 silent changes at amino acid positions $32,38,41$, $63,65,67$ ( 2 changes), 93, 114, 115, 154, 179, 186, 209, 230, and 342 just as documents were reported [27]. Moreover, the loss of 46 (Asp $\rightarrow$ Asn) was shown in 3 leukemia samples, whereas the loss of 178 (Leu $\rightarrow$ Met) was detected in 11 leukemia samples. The presence of XhoI cut site $169425 \mathrm{~g} \rightarrow \mathrm{t}(17 \mathrm{R} \rightarrow \mathrm{L})$ and the loss of a $30 \mathrm{bp}$ deletion were found in all China 1 strains (Fig. 2).

\section{China 2}

Only 2 leukemia samples belonged to the China 2 strain. This strain has 48 common changes, including 29 common changes: amino acid positions $3,13,17,32,38$, $46,51,63,65,67$ (2 changes), 82, 85, 106, 122, 126, 129, $132,144,151,154,179,192,209,212,230,309,342$, and 366; these changes are the same as those in China 1. Furthermore, other amino acid changes were found at positions $25 \mathrm{~L} \rightarrow \mathrm{M}, 33 \mathrm{~L} \rightarrow \mathrm{I}, 43 \mathrm{~V} \rightarrow \mathrm{I}, 57 \mathrm{~S} \rightarrow \mathrm{A}, 84 \mathrm{C} \rightarrow \mathrm{V}$, $91 \mathrm{~T} \rightarrow \mathrm{I}, 95 \mathrm{I} \rightarrow \mathrm{S}, 110 \mathrm{~V} \rightarrow \mathrm{L}, 115 \mathrm{G} \rightarrow \mathrm{A}, 119 \mathrm{~V} \rightarrow \mathrm{A}$, 


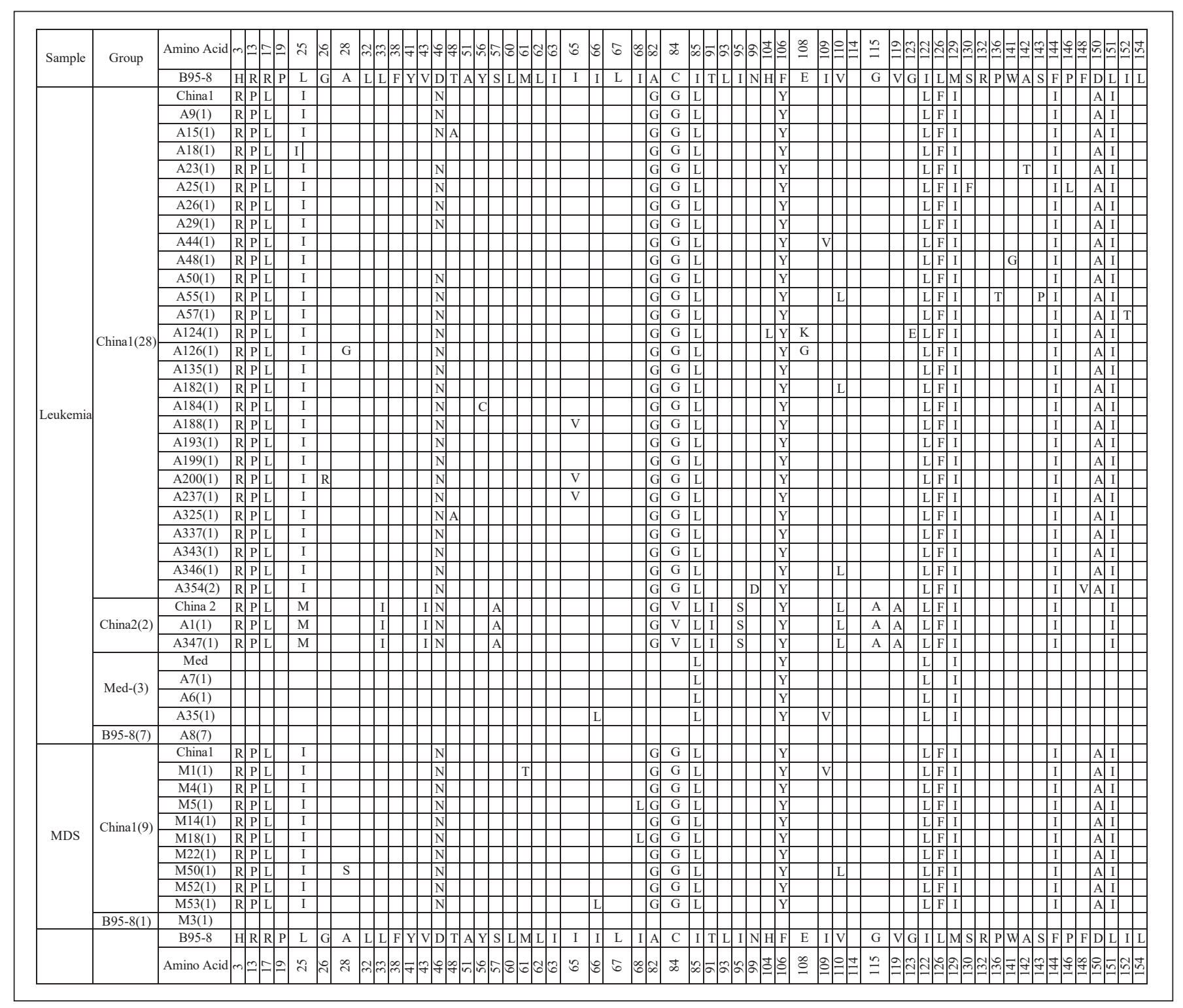

Fig. 2. LMP1 variations in leukemia and MDS in northern China (shown in two parts). The numbers across the top correspond to the amino acid positions, whereas the numbers across the second row correspond to the B95-8 nucleotide sequence. Different subtypes are noted in the second column. One sequence was chosen to represent all samples with the same sequences in the third column. The numbers in the third column refer to these representa-

$229 \mathrm{~S} \rightarrow \mathrm{T}, 245 \mathrm{P} \rightarrow \mathrm{H}, 252 \mathrm{G} \rightarrow \mathrm{D}, 331 \mathrm{G} \rightarrow \mathrm{Q}, 338 \mathrm{~L} \rightarrow$ $\mathrm{P}, 344 \mathrm{G} \rightarrow \mathrm{D}$, and $355 \mathrm{G} \rightarrow \mathrm{A}$. Two common silent changes were found at amino acid positions 60 and 320. However, both of the samples exhibited losses of $245 \mathrm{P} \rightarrow \mathrm{H}$. The sample (A1) had a loss of changes at positions 230, 245, and 334, while the sample (A347) had a loss of changes at positions $229,230,245,252$, and 334 . The presence tive isolates, and the capital letter A represents leukemia specimen, while the capital letter M represents MDS specimen. The numbers in parentheses after the specimen represent the number of specimens. The capital letters denote amino acids. Moreover, the sequence of samples in which $>2$ mutations were found is shown, and some rare sporadic mutations are not shown. LMP1, latent membrane protein 1; MDS, myelodysplastic syndrome.

(Figure continued on next page.)

of XhoI cut site $169425 \mathrm{~g} \rightarrow \mathrm{t}(17 \mathrm{R} \rightarrow \mathrm{L})$ was found in all China 2 strains, but the loss of a $30 \mathrm{bp}$ deletion was not found.

\section{Med-}

Three of the 40 leukemia samples (7.5\%) carried the Med subtype. Med includes Med+ (30 bp deficient) and 


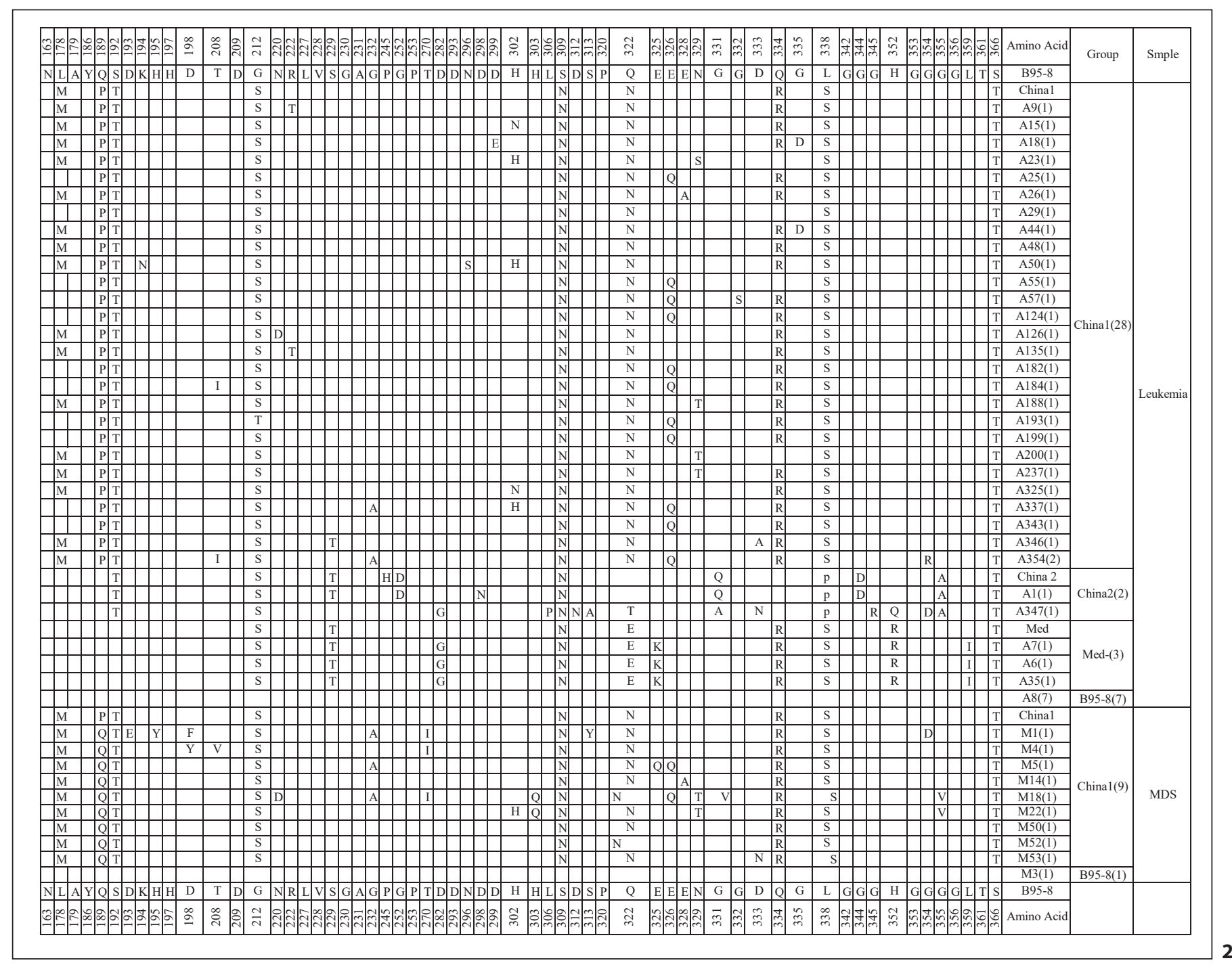

Med- (30 bp not deficient). The loss of a $30 \mathrm{bp}$ deletion was not found in all the 3 strains. So the 3 strains belonged to the Med-strain. There were fewer changes in Medthan in China 1 and China 2. Four common amino acid changes $(85,106,122$, and 129) were found in the transmembrane domain; these changes were also present in China 1 and China 2. Four common amino acid changes (at positions 212, 309, 342, and 366) were found in the cytoplasmic carboxyl-terminal domain; these changes were the same as those in China 1 and China 2. Changes at position 334 and 338 were the same as those in China 1 , whereas the change at position 229 was the same as that in China 2. The common changes at base pair positions 168357 and 168266, which led to amino acid changes at positions $322 \mathrm{Q} \rightarrow \mathrm{E}$ and $352 \mathrm{H} \rightarrow \mathrm{R}$, were unique to this strain. The silent change at 168238 was also unique to this

EBER and LMP1 Polymorphisms in the EBV-Associated Hematopathy strain. In this study, the following additional new common mutations were found: $282 \mathrm{D} \rightarrow \mathrm{G}, 325 \mathrm{E} \rightarrow \mathrm{K}$, and $359 \mathrm{~L} \rightarrow \mathrm{I}$; a silent change at 293 (168443nt A $\rightarrow$ G) was also found, whereas the rest of the changes were fully consistent with the representative Med- sequence. In addition, the presence of XhoI cut site $169425 \mathrm{~g} \rightarrow \mathrm{t}(17 \mathrm{R} \rightarrow \mathrm{L})$ was not found in all Med-strains.

The distribution of LMP1 subtypes between leukemia and MDS samples was not significantly different $(p>$ 0.05). Moreover, the distribution of LMP1 variations among leukemia, MDS, and healthy donors was not significantly different $(p>0.05)$ either.

\section{XhoI (-) Variation and Del-LMP1 Variation}

XhoI (-) variation was found in 75\% (30/40) leukemia specimens, $90 \%$ (9/10) MDS specimens, and $74.4 \%$ 
Table 3. EBER sequence variation compared to EBV type in isolates from different population groups

\begin{tabular}{llccc}
\hline EBV type & EBER & Leukemia, $n(\%)$ & MDS, $n(\%)$ & $H, n(\%)$ \\
\hline Type 1 & EB-6m & $21(53.8)$ & $8(66.6)$ & $43(75.4)$ \\
& B95-8 & $5(12.8)$ & $2(16.7)$ & 0 \\
& UG & 0 & 0 & $2(3.5)$ \\
\hline Type 2 & EB-6m & $2(5.1)$ & $2(16.7)$ & $9(15.8)$ \\
& B95-8 & $2(5.1)$ & 0 & 0 \\
\hline Type $1+2$ & EB-6m & $6(15.4)$ & 0 & $1(1.8)$ \\
\hline Total & B95-8 & $3(7.8)$ & 0 & $2(3.5)$ \\
\hline Type F & EB-6m & $30(73.2)$ & $9(81.8)$ & $83(90.2)$ \\
& EB-8m & 0 & 0 & $1(1.1)$ \\
& B95-8 & $11(26.8)$ & $2(18.2)$ & $1(1.1)$ \\
& UG & 0 & 0 & $1(1.1)$ \\
\hline Type f & EB-6m & 0 & 0 & $6(6.5)$ \\
\hline Total & & $41(100)$ & $11(100)$ & $92(100)$ \\
\hline
\end{tabular}

EBV, Epstein-Barr virus; EBER, EBV-encoded small noncoding RNA; MDS, myelodysplastic syndromes; $H$, healthy donors.

(32/43) healthy donors. While del-LMP1 variation was detected in 28 (70\%) leukemia specimens and 9 (90\%) MDS specimens as well as in $30(69.8 \%)$ healthy donors. There was no significant difference in the distribution of XhoI (-) variation as well as del-LMP1 variation among the 3 groups $(p>0.05)$. In this study, all China 1 and China 2 subtype strains lost XhoI restriction site, but all Medsubtype strains retained. Moreover, the loss of a $30 \mathrm{bp}$ deletion was found in all China 1 strains but not in China 2 strains and Med-strains.

\section{Repeat Sequence Variations of LMP1 C-Terminal}

Four perfect 11-AA (QDPDNTDDNGP) repeats were found in the C-terminal of the B95-8 strain $[22,27,34]$, with a 5-AA (HDPLP) insertion after the second 11-AA (QDPDNTDDNGP). The 5-AA insertion was absent in the other subtypes. The copy number of the repeat element varied from 3 to 8 copies independent of the strain. Most China 1 strains had 4, 5, or 6 repeats. Both China 2 strains carried 4 repeats. Two Med- strains carried 4 repeats, and $1 \mathrm{Med}-$ strain had 5 repeats. China 1 had a high-level repeat number (5-8 copies) of 53.6\% (15/28), which was higher than that in the China 2 and Med- subtypes ( $0 / 2$ and $1 / 3$, respectively).
Table 4. LMP1 sequence variation compared to EBV type in isolates from different population groups

\begin{tabular}{lllll}
\hline EBV type & $\begin{array}{l}\text { LMP1 } \\
(30 \text { bp deletion })\end{array}$ & $\begin{array}{l}\text { Leukemia, } \\
(\%)\end{array}$ & $\begin{array}{l}\text { MDS, } \\
n(\%)\end{array}$ & $\begin{array}{l}H, \\
n(\%)\end{array}$ \\
\hline Type 1 & Deleted & $21(55.3)$ & $9(90)$ & $27(71.1)$ \\
& Retained & $7(18.4)$ & $1(10)$ & $2(5.2)$ \\
\hline Type 2 & Deleted & $2(5.3)$ & 0 & $1(2.6)$ \\
& Retained & $1(2.6)$ & 0 & $8(21.1)$ \\
\hline Type 1+2 & Deleted & $3(7.9)$ & 0 & 0 \\
& Retained & $4(10.5)$ & 0 & 0 \\
\hline Total & & $38(100)$ & $10(100)$ & $38(100)$ \\
\hline Type F & Deleted & $27(69.2)$ & $8(80)$ & $29(69)$ \\
& Retained & $11(28.2)$ & $1(10)$ & $11(26.2)$ \\
\hline Type f & Deleted & $1(2.6)$ & $1(10)$ & $1(2.4)$ \\
& Retained & 0 & 0 & $1(2.4)$ \\
\hline Total & & $39(100)$ & $10(100)$ & $42(100)$ \\
\hline
\end{tabular}

EBV, Epstein-Barr virus; LMP1, latent membrane protein 1; MDS, myelodysplastic syndromes; $H$, healthy donors.

\section{EBER Sequence Variations versus EBV Types in Leukemia and MDS Samples}

The relationship between EBER subtypes and EBV types in leukemia, MDS, and healthy donors from Northern China is shown in Table 3. As we found in our previous report, the EBV genotype 1 in MDS showed heterogeneity in terms of EBER variation. EB-6m $(66.6 \%, 8 / 12)$ and $\mathrm{B} 95-8(16.7 \%, 2 / 12)$ variants were all demonstrated in type $1 \mathrm{EBV}$ strain. All the type 2 strains carried the EB$6 \mathrm{~m}$ variant. However, the EB-6m and B95- 8 variants were all found in type $1 \mathrm{EBV}$ strain, type $2 \mathrm{EBV}$ strain, and type $1+2 \mathrm{EBV}$ strain in leukemia. In our previous research, the EB-6m, EB-8m, and EB-10m variants were all found in type $1 \mathrm{EBV}$ strain. Compared with our previous studies, we speculate that EB-8m and EB-10m variants might only be found in type $1 \mathrm{EBV}$ strain. Therefore, the EBV genotype 1 in leukemia also showed heterogeneity in terms of EBER variation. Among these 3 groups, EB-6m variant was the most common isolate in type $1 \mathrm{EBV}$ strain, type 2 EBV strain, and type $1+2$ EBV strain. Twenty-one of $39(53.8 \%)$ leukemia, 8 of 12 (66.6\%) MDS, and 43 of 57 (75.4\%) healthy donors belonged to the type 1-EB-6m. There was no significant difference in the distribution of the type 1-EB-6m among the 3 groups $(p>0.05)$.

Five independent combinational genotypes between BamHI F genotypes and EBER variants are characterized in Table 3. The type F-EB-6m was detected in $73.2 \%$ 
(30/41) of leukemia, $81.8 \%$ of (9/11) MDS, and $90.2 \%$ of (83/92) healthy donors, while the type F-B95-8 was detected in $26.8 \%$ (11/41) of leukemia, $18.2 \%$ (2/11) of MDS, and $1.1 \%$ of $(1 / 92)$ healthy donors. The type F-EB- $6 \mathrm{~m}$ was the main EBV isolate among these 3 groups. In addition, the distribution of the type F-EB-6m among leukemia, MDS, and healthy donors was not significantly different $(p>0.05)$ either.

\section{LMP1 Sequence Variations versus EBV Types in \\ Leukemia and MDS Samples}

The linkage between LMP1 subtypes and EBV types in these 3 groups is shown in Table 4 . As it is shown in Table 4 , the type 1-LMP1-deletion variant was the most common one among these 3 groups. Twenty-one of 38 (55.3\%) leukemia, 9 of 10 (90\%) MDS, and 27 of 38 (71.1\%) healthy donors carried the type 1-LMP1-deletion variant. The distribution of the type 1-LMP1-deletion among these 3 groups was not significantly different $(p>0.05)$.

The LMP1-deletion variant $(69.2 \%, 27 / 39)$ and the LMP1-retention variant $(28.2 \%, 11 / 39)$ were demonstrated in the type F EBV strain in leukemia, while 8 (80\%) LMP1-deletion variant and 1 (10\%) LMP1-retention variant were characterized in the type F EBV strain in MDS. Among them, the type F-LMP1-deletion variant was the dominant one, which was found in 27 (69.2\%) leukemia, 8 (80\%) MDS, and 29 (69\%) healthy donors. There was no significant difference in the distribution of the type F-LMP1-deletion variant among these 3 groups $(p>0.05)$.

\section{Discussion}

EBV may play a role in the pathogenesis of leukemia and MDS [9, 12, 35]. In our studies, EBV-DNA sequences were found in 88 of the 322 (27.3\%) leukemia samples and in 35 of the 106 MDS samples (33\%). The frequencies of EBV infection, as described here, were similar to those reported in AL patients (34.6\%) and higher than those found in healthy controls $(5.4 \%, 2 / 37)$ [8].

In this study, type $1 \mathrm{EBV}$ and type F EBV were the main EBV subtypes in leukemia and MDS in Shandong. These results are consistent with the results of our previous report on other EBV-positive specimens (e.g., gastric carcinoma, NPC, lymphoma, and healthy donors) [28, 30 ], as well as in most other parts of the world. Genotype $1 / 2$ frequency differs dramatically across the geographic regions of the world. Genotype 1 is the predominant strain in most regions, whereas genotype 2 is predomi-

EBER and LMP1 Polymorphisms in the EBV-Associated Hematopathy nant only in Central Africa and New Guinea [36, 37]. Type F EBV is the main genotype except endemic area of NPC in Southern China, while type f EBV is more common in Southern China [30]. The distribution of type $1 / 2$ or F/f in leukemia and MDS samples was similar to other groups in Northern China and reflected region restriction of EBV subtypes.

In the past studies, 4 types of EBER genotypes were found: EB-6m, EB-8m, EB-10m, and B95-8. EB-6m was the most common variant, including 13 (81.3\%) HD (Hodgkin's disease) cases and 19 (79.2\%) PTLD (posttransplant lymphoproliferative disorders) in America [38, 39], 31 (75.6\%) benign and malignant diseases in Germany [40], 14 (93.3\%) HIV-infected individuals, 7 (63.6\%) healthy human donors in Italy [41], and 18 (85.7\%) NPC tissues in South Africa [42]. In our previous study, EB-6m was the most common type in gastric carcinoma, NPC, lymphoma, and healthy donors in Northern China, a non-endemic NPC area [28, 31]. However, EB-8m was more common in endemic NPC cases $(82.0 \%, 41 / 50)$ than in non-endemic NPC cases $(33.7 \%, 32 / 95)$, and it was also more frequent in healthy donors from endemic area $(32.4 \%, 24 / 74)$ than healthy donors from non-endemic area $(1.1 \%, 1 / 92)$. More importantly, the EB-8m was more prevalent in NPC cases than healthy donors in both areas [30]. These results suggested an association between NPC and EBV EB-8m isolates.

EB-6m was also the most common isolate in leukemia and MDS in Northern China, just as in background populations in the same area (gastric carcinoma, NPC, lymphoma, and healthy donors), which may reflect that EBER variations may not be associated with the pathogenesis of hematopathy. The significance of lower frequency of EB-6m in leukemia and MDS than in healthy people needs further study to confirm.

LMP1 was divided into 7 distinct sequence patterns or strains on the basis of the amino acid (aa) changes of the carboxyl-terminal sequence (aa187-386) from different geographical origins: B95-8, China 1, China 2, China 3, Med (Mediterranean), NC (North Carolina), and Alaskan [27]. According to the data summarized by Edwards et al. [27] and the study of Banko et al. [34], China 1, B95-8, Med, and NC subtypes were detected in 67 cases of European lymphomas, most of which were China 1 and B95-8. The detection rate of China 1 subtype was $45 \%$. China 1, China 2, B95-8, Med, NC, and Alaskan subtypes were detected in 56 cases of American lymphoma, and China 1 and B95-8 were more common. The detection rate of China 1 subtype was about 
45\%. In South America, China 1, Med-, Alaskan, B958 , China 2, and China 3 were detected in 62 cases of lymphoma according to the data of Guiretti et al. [43] and Lorenzetti et al. [44], and China 1 and Med were the dominant subtype. Compared with the data of lymphoma in different regions, the variation of LMP1 showed a regional distribution trend.

In our leukemia and MDS samples, the most dominant LMP1 strain was China 1, a difference which was not significant $(p>0.05)$. The result was consistent with previous report, where China 1 is predominant in East Asia, whereas NPC is endemic in healthy donors from northern China $[29,45]$. Therefore, the role of LMP1 variation in the pathogenesis of leukemia and MDS may be the same as that in other tumors. The LMP1 gene is considered a viral oncogene because of its ability to transform rodent fibroblasts and human keratinocytes, induce lymphoma and hyperplasia in transgenic mice, and induce the expression of epidermal growth factor receptors [34, 46]. These findings strongly suggest that LMP1 plays a crucial role in the development of EBV-related carcinomas. Therefore, it is necessary to study the function of China 1 subtype and EBV strain with China 1 subtype and to explore the effect of the variation on carcinogenic potential.

XhoI (-) mutation and del-LMP1 mutation were once considered as a specific tumor marker for NPC in China according to the high detection of the 2 variants [24, 47]. Many studies have detected XhoI (-) variation and delLMP1 variation in samples from different regions of the world. The relationship between the 2 variants and EBVrelated diseases is also a controversial topic. In our study, the XhoI (-) variation was found in all China 1 and China 2 subtype strains but not in Med- subtype strains. Moreover, the del-LMP1 mutation was found in all China 1 strains but not in China 2 and Med-strains. Therefore, detection of the 2 mutations does not fully reflect the variation of LMP1. In a word, in order to fully and truly analyze the variation of LMP1, we should detect and analyze the whole LMP1 sequence.

In our study, the type $1 \mathrm{EBV}$ strain was heterogeneous in terms of EBER variation in leukemia and MDS. The result was consistent with the result of our previous report $[28,31]$. Therefore, EBER locus can be useful for the identification of different EBV isolates, but it is not reliable as a marker for EBV type. The prototype $\mathrm{F}$ isolates tended to be mainly correlated with the EB-6m variant, and the type F-EB-6m was the main EBV isolate among these 3 groups. The result was consistent with that of previous research [30].
The predominance of EBV type 1 and its association with the deletion variant of the LMP1 gene in leukemia and MDS samples is in agreement with other studies on Chinese NPC in Hong Kong [48]. In addition, the type F-LMP1-deletion variant was the dominant one in our leukemia and MDS samples. Therefore, it is necessary to study the relationship between LMP1-deletion variant and EBV types in EBV-associated diseases from different regions. Moreover, the investigation of multiple polymorphic sites in the EBV genome might be contributed to a reliable assessment of EBV-related diseases.

In conclusion, EBV genotypes 1 and $\mathrm{F}$ were the dominant ones associated with leukemia and MDS in Northern China. The distribution of the EBV genotypes was geographically restricted. The distribution of EBER and LMP1 subtypes in leukemia and MDS was similar to that in the background population in Northern China, which means that these subtypes may be only region restricted and not associated with leukemia and MDS pathogenesis.

\section{Statement of Ethics}

All experiments were performed in compliance with relevant laws and institutional guidelines and in accordance with the ethical standards of the Declaration of Helsinki. This study was approved by the Medical Ethics Committee at the Medical College of Qingdao University (Ref. No. QYFY WZLL 25848). Informed consents were obtained from all individual participants included in the study.

\section{Conflict of Interest Statement}

The authors have no conflicts of interest to declare.

\section{Funding Sources}

This work was supported by the National Natural Science Foundation of China (NSFC 81571995) and the Natural Science Foundation of Shandong Province (ZR2017BH106).

\section{Author Contributions}

Z.-G.Z. and B.L. conceived the project and designed experiments. P.L. mainly obtained specimens from the Affiliated Hospital of Qingdao University. H.Y.W. mainly conducted the experiments. L.S. and W.L. participated in performance of the experiments and the drafting of the manuscript. All authors read and approved the final manuscript. 


\section{References}

1 Klein G. Tumor associations of EBV: historical perspectives. Curr Top Microbiol Immunol. 2015;390(Pt 1):17-22.

2 Tsang CM, Tsao SW. The role of Epstein-Barr virus infection in the pathogenesis of nasopharyngeal carcinoma. Virol Sin. 2015;30(2): 107-21.

3 Shinozaki-Ushiku A, Kunita A, Fukayama M. Update on Epstein-Barr virus and gastric cancer (review). Int J Oncol. 2015;46(4):1421-34.

4 Auerbach A, Aguilera NS. Epstein-Barr virus (EBV)-associated lymphoid lesions of the head and neck. Semin Diagn Pathol. 2015; 32(1):12-22.

5 Tsao SW, Tsang CM, To KF, Lo KW. The role of Epstein-Barr virus in epithelial malignancies. J Pathol. 2015;235(2):323-33.

6 Klein E, Nagy N. Restricted expression of EBV encoded proteins in in vitro infected CLL cells. Semin Cancer Biol. 2010;20(6):410-5.

7 Ateyah ME, Hashem ME, Abdelsalam M. Epstein-Barr virus and regulatory $\mathrm{T}$ cells in Egyptian paediatric patients with acute B lymphoblastic leukaemia. J Clin Pathol. 2017; 70(2):120-5.

8 Guan H, Miao H, Ma N, Lu W, Luo B. Correlations between Epstein-Barr virus and acute leukemia. J Med Virol. 2017;89(8): 1453-60.

9 Ahmed HG, Osman SI, Ashankyty IM. Incidence of Epstein-Barr virus in pediatric leukemia in the Sudan. Clin Lymphoma Myeloma Leuk. 2012;12(2):127-31.

10 Mahjour SB, Ghaffarpasand F, Fattahi MJ, Ghaderi A, Fotouhi Ghiam A, Karimi M. Seroprevalence of human herpes simplex, hepatitis B and Epstein-Barr viruses in children with acute lymphoblastic leukemia in southern Iran. Pathol Oncol Res. 2010;16(4):579_ 82.

11 Sehgal S, Mujtaba S, Gupta D, Aggarwal R, Marwaha RK. High incidence of Epstein Barr virus infection in childhood acute lymphocytic leukemia: a preliminary study. Indian J Pathol Microbiol. 2010;53(1):63-7.

12 Dolcetti R, Carbone A. Epstein-Barr virus infection and chronic lymphocytic leukemia: a possible progression factor? Infect Agents Cancer. 2010;5:22.

13 Ferrajoli A, Ivan C, Ciccone M, Shimizu M, Kita Y, Ohtsuka M, et al. Epstein-Barr virus MicroRNAs are expressed in patients with chronic lymphocytic leukemia and correlate with overall survival. EBioMedicine. 2015; 2(6):572-82.

14 Visco C, Falisi E, Young KH, Pascarella M, Perbellini O, Carli G, et al. Epstein-Barr virus DNA load in chronic lymphocytic leukemia is an independent predictor of clinical course and survival. Oncotarget. 2015;6(21):18653-63.

15 Sample J, Young L, Martin B, Chatman T, Kieff E, Rickinson A, et al. Epstein-Barr virus types 1 and 2 differ in their EBNA-3A, EBNA3B, and EBNA-3C genes. J Virol. 1990;64(9): 4084-92.
16 Yao QY, Tierney RJ, Croom-Carter D, Dukers D, Cooper GM, Ellis CJ, et al. Frequency of multiple Epstein-Barr virus infections in Tcell-immunocompromised individuals. J Virol. 1996;70(8):4884-94.

17 Midgley RS, Blake NW, Yao QY, Croom-Carter D, Cheung ST, Leung SF, et al. Novel intertypic recombinants of Epstein-Barr virus in the Chinese population. J Virol. 2000;74(3):1544-8.

18 Lung ML, Chang RS, Jones JH. Genetic polymorphism of natural Epstein-Barr virus isolates from infectious mononucleosis patients and healthy carriers. J Virol. 1988;62(10): 3862-6.

19 Chen JN, Ding YG, Feng ZY, Li HG, He D, Du $\mathrm{H}$, et al. Association of distinctive EpsteinBarr virus variants with gastric carcinoma in Guangzhou, Southern China. J Med Virol. 2010;82(4):658-67.

20 Chen WG, Chen YY, Bacchi MM, Bacchi CE, Alvarenga M, Weiss LM. Genotyping of Epstein-Barr virus in Brazilian Burkitt's lymphoma and reactive lymphoid tissue. Type A with a high prevalence of deletions within the latent membrane protein gene. Am J Pathol. 1996;148(1):17-23.

21 Yao QY, Rowe M, Martin B, Young LS, Rickinson $\mathrm{AB}$. The Epstein-Barr virus carrier state: dominance of a single growth-transforming isolate in the blood and in the oropharynx of healthy virus carriers. J Gen Virol. 1991;72(Pt 7):1579.

22 Kieser A, Sterz KR. The latent membrane protein 1 (LMP1). Curr Top Microbiol Immunol. 2015;391:119-49.

23 Eliopoulos AG, Young LS. LMP1 structure and signal transduction. Semin Cancer Biol. 2001;11(6):435-44.

$24 \mathrm{Hu}$ LF, Zabarovsky ER, Chen F, Cao SL, Ernberg I, Klein G, et al. Isolation and sequencing of the Epstein-Barr virus BNLF-1 gene (LMP1) from a Chinese nasopharyngeal carcinoma. J Gen Virol. 1991;72(Pt 10):2399_ 409.

25 Miller WE, Edwards RH, Walling DM, RaabTraub N. Sequence variation in the EpsteinBarr virus latent membrane protein 1. J Gen Virol. 1994;75(Pt 10):2729-40.

26 Tacyildiz N, Cavdar AO, Ertem U, Oksal A, Kutluay L, Uluoglu O, et al. Unusually high frequency of a 69-bp deletion within the carboxy terminus of the LMP-1 oncogene of Epstein-Barr virus detected in Burkitt's lymphoma of Turkish children. Leukemia. 1998; 12(11):1796-805.

27 Edwards RH, Seillier-Moiseiwitsch F, RaabTraub N. Signature amino acid changes in latent membrane protein 1 distinguish EpsteinBarr virus strains. Virology. 1999;261(1):7995.

28 Zhao Z, Sun L, Liu S, Shu J, Luo B. Characterization of Epstein-Barr virus-encoded small RNA gene variations in virus associated lymphomas in Northern China. Arch Virol. 2017; 162(6):1609-16.
29 Wang Y, Kanai K, Satoh Y, Luo B, Sairenji T. Carboxyl-terminal sequence variation of latent membrane protein 1 gene in EpsteinBarr virus-associated gastric carcinomas from Eastern China and Japan. Intervirology. 2007; 50(3):229-36.

30 Shen ZC, Luo B, Chen JN, Chao Y, Shao CK, Liu QQ, et al. High prevalence of the EBER variant EB-8m in endemic nasopharyngeal carcinomas. PLoS One. 2015;10(3): e0121420.

31 Wang Y, Zhang X, Chao Y, Jia Y, Xing X, Luo B. New variations of Epstein-Barr virus-encoded small RNA genes in nasopharyngeal carcinomas, gastric carcinomas, and healthy donors in northern China. J Med Virol. 2010; 82(5):829-36.

32 Borisch B, Finke J, Hennig I, Delacrétaz F, Schneider J, Heitz PU, et al. Distribution and localization of Epstein-Barr virus subtypes A and B in AIDS-related lymphomas and lymphatic tissue of HIV-positive patients. J Pathol. 1992;168(2):229-36.

33 Cui Y, Wang Y, Liu X, Chao Y, Xing X, Zhao C, et al. Genotypic analysis of Epstein-Barr virus isolates associated with nasopharyngeal carcinoma in Northern China. Intervirology. 2011;54(3):131-8.

34 Banko A, Lazarevic I, Cupic M, Stevanovic G, Boricic I, Jovanovic T. Carboxy-terminal sequence variation of LMP1 gene in EpsteinBarr-virus-associated mononucleosis and tumors from Serbian patients. J Med Virol. 2012;84(4):632-42.

35 Borze I, Scheinin I, Siitonen S, Elonen E, Juvonen E, Knuutila S. miRNA expression profiles in myelodysplastic syndromes reveal Epstein-Barr virus miR-BART13 dysregulation. Leuk Lymphoma. 2011;52(8):1567-73.

36 Chang CM, Yu KJ, Mbulaiteye SM, Hildesheim A, Bhatia K. The extent of genetic diversity of Epstein-Barr virus and its geographic and disease patterns: a need for reappraisal. Virus Res. 2009;143(2):20921.

37 Gratama JW, Ernberg I. Molecular epidemiology of Epstein-Barr virus infection. Adv Cancer Res. 1995;67:197-255.

38 Frank D, Cesarman E, Liu YF, Michler RE, Knowles DM. Posttransplantation lymphoproliferative disorders frequently contain type $A$ and not type B Epstein-Barr virus. Blood. 1995;85(5):1396-403.

39 Lin JC, Lin SC, De BK, Chan WP, Evatt BL, Chan WC. Precision of genotyping of Epstein-Barr virus by polymerase chain reaction using three gene loci (EBNA-2, EBNA-3C, and EBER): predominance of type A virus associated with Hodgkin's disease. Blood. 1993; 81(12):3372-81.

40 Schuster V, Ott G, Seidenspinner S, Kreth HW. Common Epstein-Barr virus (EBV) type- 1 variant strains in both malignant and benign EBV-associated disorders. Blood. 1996;87(4):1579. 
41 Menin C, Ometto L, Veronesi A, Roncella S, Corneo B, Montagna M, et al. Analysis of Epstein-Barr virus (EBV) type and variant in spontaneous lymphoblastoid cells and $\mathrm{Hu}-$ SCID mouse tumours. Mol Cell Probes. 1996; 10:453.

42 Janse vRE, van Heerden WF, Robson BA, Swart TJ, Engelbrecht S. Epstein-Barr virus strain characterisation in South African patients with nasopharyngeal carcinomas. Anticancer Res. 2000;20:1953-7.

43 Guiretti DM, Chabay PA, Valva P, Stefanoff CG, Barros MH, De Matteo E, et al. Structural variability of the carboxy-terminus of Epstein-Barr virus encoded latent membrane protein 1 gene in Hodgkin's lymphomas. J Med Virol. 2007;79(11):1730-22.
44 Lorenzetti MA, Gantuz M, Altcheh J, De Matteo E, Chabay PA, Preciado MV. Distinctive Epstein-Barr virus variants associated with benign and malignant pediatric pathologies: LMP1 sequence characterization and linkage with other viral gene polymorphisms. J Clin Microbiol. 2012;50(3):609-18.

45 Sung NS, Edwards RH, Seillier-Moiseiwitsch F, Perkins AG, Zeng Y, Raab-Traub N. Epstein-Barr virus strain variation in nasopharyngeal carcinoma from the endemic and non-endemic regions of China. Int J Cancer. 1998;76(2):207-15.
46 Thompson MP, Kurzrock R. Epstein-Barr virus and cancer. Clin Cancer Res. 2004;10(3): $803-21$.

47 Chen ML, Tsai CN, Liang CL, Shu CH, Huang CR, Sulitzeanu D, et al. Cloning and characterization of the latent membrane protein (LMP) of a specific Epstein-Barr virus variant derived from the nasopharyngeal carcinoma in the Taiwanese population. Oncogene. 1992;7(11):2131-40.

48 Cheung ST, Leung SF, Lo KW, Chiu KW, Tam JS, Fok TF, et al. Specific latent membrane protein 1 gene sequences in type 1 and type 2 Epstein-Barr virus from nasopharyngeal carcinoma in Hong Kong. Int J Cancer. 1998;76(3):399-406. 